\title{
On local isometric immersions of the spaces of negative constant curvature into the euclidean spaces
}

\author{
By
}

Eiji KanEDa

(Received Feb. 21, 1978)

\section{Introduction}

In his paper [9], T. Otsuki obtained an estimate for the lower bound of the dimensions of the euclidean spaces in which a space of negative constant curvature can be locally isometrically immersed. He proved that any space of negative constant curvature of dimension $n$ cannot be isometrically immersed into the euclidean space $\boldsymbol{R}^{2 n-2 \text {. }}$

The main purpose of this paper is to show the following

Theorem 5.2. Any space of negative constant curvature of dimension $n$ can be locally isometrically immersed into the euclidean space $\boldsymbol{R}^{2 n-1}$.

We wish to prove this theorem by a method based on the theory of non-linear partial differential equations established by M. Kuranishi [8] and H. Goldschmidt [4].

Let $(M, g)$ be an $n$-dimensional Riemannian manifold. Let $P$ be the differential equation of isometric immersions of $(M, g)$ into the euclidean space $\boldsymbol{R}^{m}$ with $m \geqq n$, which is a system of non-linear parital differential equations of order 1 . Adjoining to this system $P$ the equation of Gauss-Weingarten which is obtained by differentiating the equation of isometric immersions, we obtain the system $P^{(1)}$ of order 2 , the first prolongation of $P$. Similarly adjoining to the system $P^{(1)}$ the equation which is obtained by differentiating the equation of Gauss-Weingarten, we obtain the system of order 3 , the second prolongation of $P$. A formal solution of order 1 can be always be extended to a formal solution of order 2 , while a formal solution of order 2 cannot be necessarily extended to a formal solution of order 3 . There exists an obstruction to extending the formal solutions of order 2 to the formal solutions of order 3, which is called the equation of Gauss.

Recently J. Gasqui [3] gave a new proof of the famous theorem of JanetCartan, showing that the system $Q$ which is obtained by adjoining the equation of Gauss to the system $P^{(1)}$ forms an involutive system under the assumption $m \geqq$ $\frac{1}{2} n(n+1)$. Another proof of the theorem of Janet-Cartan from a somewhat different viewpoint was delivered by $\mathrm{N}$. Tanaka in his lecture at Kyoto University before 
Gasqui's paper was published (cf. [6]). He proved that the symbol $\mathfrak{q}$ of the system $Q$ is isomorphic to the second prolongation $\mathfrak{h}^{(2)}$ of the symbol $\mathfrak{h}$ of the linear operator $L$ which was first introduced by himself (see [10]), and proved that if $m \geqq \frac{1}{2} n(n+1)$, then at each generic point of $Q$ the symbol $\mathfrak{h}$ is involutive. Here we remark that the vanishing of the Spencer cohomology group of the symbol $\mathfrak{h}$ plays an important role in his proof of the theorem of Janet-Cartan.

In this paper we need to investigate the system $Q$ under the assumption $m<\frac{1}{2} n(n+1)$. Unfortunately the symbol $\mathfrak{h}$ cannot be involutive in this case. Hence the method developed by N. Tanaka cannot be applied to our problem. However, by letting $(M, g)$ be a space of negative constant curvature, we can prove the following

Theorem 5.1. If $m=2 n-1$, then there exists an open fibered submanifold $\pi_{1}^{2}: P_{\#}^{(1)}$ $\rightarrow P$ of the vector bundle $\pi_{1}^{2}: P^{(1)} \rightarrow P$ such that the intersection $Q_{\sharp}=Q \cap P^{(1)}$ forms an involutive differential equation.

Theorem 5.2 cited above now follows from Theorem 5.1.

Following the formulations given by $\mathrm{N}$. Tanaka, we recall in $\S 1$ the differential equations $P, P^{(1)}$, etc. In $\S 2$, we define the formal Gaussian variety with respect to a curvature like tensor. $\S 3$ and $\S 4$ are devoted to the proof of Theorem 3.1 that describes the properties of the formal Gaussian variety with respect to a curvature like tensor of negative constant curvature. Finally in $\S 5$ we prove Theorem 5.1.

The author would like to express his gratitude to Professor N. Tanaka for his kind advices and constant encouragements.

\section{§1. The differential equations associated with isometric immersions}

Let $M$ be an $n$-dimensional differentiable manifold ${ }^{(*)}$ and let $T=T(M)$ (resp. $T^{*}=T^{*}(M)$ ) be the tangent (resp. cotangent) bundle of $M$. By $\otimes^{k} T^{*}$ (resp. $S^{k} T^{*}$ ), we mean the bundle of $k$-tensors (resp. symmetric $k$-tensors) on $M$. Let $g$ be a Riemannian metric on $M$. We denote by $\nabla$ (resp. $R$ ) the covariant differentiation (resp. the curvature tensor) associated with the Riemannian connectoin on $M$ induced from $g$.

Let $\boldsymbol{R}^{m}$ be the $m$-dimensional euclidean space with $m \geqq n$ and $\langle$,$\rangle be the$ standard inner product of $\boldsymbol{R}^{m}$. We denote by $\bar{g}$ the cannonical Riemannian metric of $\boldsymbol{R}^{m}$ induced from $\langle$,$\rangle .$

By definition an isometric immersion $f$ of the Riemannian manifold $(M, g)$ into the euclidean space $\boldsymbol{R}^{m}$ is an immersion of $M$ into $\boldsymbol{R}^{m}$ which is a solution of the equation

$$
f^{*} \bar{g}=g
$$

where $f^{*} g$ stands for the Riemannian metric on $M$ induced from $g$ by $f$.

Let $f$ be an isometric immersion of $(M, g)$ into $R^{m}$. Then at each $p \in M$, we have the following equalities (cf. Proposition 2 of Appendix in [6]):

(*) Throughout this paper we shall assume the differentiability of class $C^{\infty}$. 


$$
\begin{gathered}
\left\langle\nabla_{x} f, \nabla_{y} f\right\rangle=g(x, y), \\
\left\langle\nabla_{z} \nabla_{x} f, \nabla_{y} f\right\rangle=0, \\
\left\langle\nabla_{u} \nabla_{z} \nabla_{x} f, \nabla_{y} f\right\rangle+\left\langle\nabla_{z} \nabla_{x} f, \nabla_{u} \nabla_{y} f\right\rangle=0, \\
\left\langle\nabla_{z} \nabla_{x} f, \nabla_{u} \nabla_{y} f\right\rangle-\left\langle\nabla_{u} \nabla_{x} f, \nabla_{z} \nabla_{y} f\right\rangle=-g(R(z, u) x, y), \\
\left\langle\nabla_{v} \nabla_{z} \nabla_{x} f, \nabla_{u} \nabla_{y} f\right\rangle+\left\langle\nabla_{z} \nabla_{x} f, \nabla_{v} \nabla_{u} \nabla_{y} f\right\rangle-\left\langle\nabla_{v} \nabla_{u} \nabla_{x} f, \nabla_{z} \nabla_{y} f\right\rangle \\
-\left\langle\nabla_{u} \nabla_{x} f, \nabla_{v} \nabla_{z} \nabla_{y} f\right\rangle=-g\left(\nabla_{v} R(z, u) x, y\right) \quad \text { for } x, y, z, u, v \in T_{p} .
\end{gathered}
$$

Classically the equation (1.2) (resp. (1.4)) is called the equation of Gauss-Weingarten (resp. the equation of Gauss).

Let $J^{k}(M, m)$ be the vector bundle of all $k$-jets of local differentiable maps of $M$ into $\boldsymbol{R}^{m}$. By $\pi_{k-1}^{k}$ we mean the canonical projection of $J^{k}(M, m)$ onto $J^{k-1}(M, m)$ and by $\pi_{-1}^{k}$ the source map of $J^{k}(M, m)$ onto $M$. As usual the set of formal solutions of order $k$ are represented by a subvariety of $J^{k}(M, m)$. We denote by $P$ the subvariety of $J^{1}(M, m)$ composed of all 1-jets satisfying the equation (1.1) and by $P^{(1)}$ the subvariety of $J^{2}(M, m)$ composed of all 2-jets satisfying the system of equations (1.1) and (1.2). We also denote by $Q$ the subvariety of $J^{2}(M, m)$ composed of all 2-jets satisfying the system of equations (1.1), (1.2) and (1.4) and by $Q^{(1)}$ the subvariety of $J^{3}(M, m)$ composed of all 3-jets satisfying the system of equations (1.1) (1.5). Note that $P^{(1)}$ (resp. $Q^{(1)}$ ) is the first prolongation of $P$ (resp. $Q$ ) in the usual sense.

We now give the explicit expressions of the varieties $P, P^{(1)}, Q$ and $Q^{(1)}$.

Let $\otimes{ }^{k} T^{*} \otimes R^{m}$ be the vector bundle of all $\boldsymbol{R}^{m}$-valued $k$-tensors on $M$. Let us set $T^{k}(M, m)=\sum_{i=0}^{k} \otimes{ }^{i} T^{*} \otimes R^{m}$. We shall represent every element $\omega \in T^{k}(M, m)$ by $\omega=\left(p ; \omega_{0}, \omega_{1}, \cdots, \omega_{k}\right)$, where $p$ is the origin of $\omega$ and $\omega_{i} \in \otimes^{i} T_{p}^{*} \otimes \boldsymbol{R}^{m}(i=0,1, \cdots$, $k$ ). As in Appendix in [6], we shall consider the vector bundle $J^{k}(M, m)$ as a subbundle of the vector bundle $T^{k}(M, m)$. We have $J^{0}(M, m)=T^{0}(M, m)$ and $J^{1}(M, m)$ $=T^{1}(M, m)$. The bundles $J^{2}(M, m)$ and $J^{3}(M, m)$ can be characterized as follows: $J^{2}(M, m)$ consists of all $\left(p ; \omega_{0}, \omega_{1}, \omega_{2}\right) \in T^{2}(M, m)$ such that

$$
\omega_{2}(x, y)=\omega_{2}(y, x) \quad \text { for any } x, y \in T_{p} .
$$

$J^{3}(M, m)$ consists of all $\left(p ; \omega_{0}, \omega_{1}, \omega_{2}, \omega_{3}\right) \in T^{3}(M, m)$ such that

$$
\begin{aligned}
& \left(p ; \omega_{0}, \omega_{1}, \omega_{2}\right) \in J^{2}(M, m) ; \\
& \omega_{3}(x, y, z)=\omega_{3}(y, x, z)-\omega_{1}(R(x, y) z), \\
& \omega_{3}(x, y, z)=\omega_{3}(x, z, y) \quad \text { for any } x, y, z \in T_{p} .
\end{aligned}
$$

These being prepared, we give the explicit expressions of the varieties $P, P^{(1)}, Q$ and $Q^{(1)}$.

The variety $P$ is composed of all $\left(p ; \omega_{0}, \omega_{1}\right) \in J^{1}(M, m)$ satisfying

$$
\left\langle\omega_{1}(x), \omega_{1}(y)\right\rangle=g(x, y) \quad \text { for any } x, y \in T_{p} .
$$


It is easily observed that $P$ is a fibered submanifold of $\pi_{-1}^{1}: J^{1}(M, m) \rightarrow M$. Let $\alpha=$ $\left(p ; \omega_{0}, \omega_{1}\right) \in P$. We mean by $N_{\alpha}$ the orthogonal complement of the subspace $\omega_{1}\left(T_{p}\right)$ in $\boldsymbol{R}^{m}$. Then the union $N=\bigcup_{\alpha \in P} N_{\alpha}$ forms a vector bundle over $P$.

The variety $P^{(1)}$ is composed of all $\left(P ; \omega_{0}, \omega_{1}, \omega_{2}\right) \in J^{2}(M, m)$ satisfying $\left(p ; \omega_{0}\right.$, $\left.\omega_{1}\right) \in P$ and

$$
\left\langle\omega_{2}(z, x), \omega_{1}(y)\right\rangle=0 \quad \text { for any } x, y, z \in T_{p} .
$$

We can easily see that $P^{(1)}$ forms a vector bundle over $P$ which is isomorphic to the vector bundle $S^{2} T^{*} \otimes_{P} N$.

Analogously the variety $Q$ consists of all $\left(p ; \omega_{0}, \omega_{1}, \omega_{2}\right) \in P^{(1)}$ satisfying

$$
\begin{aligned}
\left\langle\omega_{2}(z, x), \omega_{2}(u, y)\right\rangle-\left\langle\omega_{2}(u, x), \omega_{2}(z, y)\right\rangle=- & g(R(z, u) x, y) \\
& \text { for any } x, y, z, u \in T_{p},
\end{aligned}
$$

and the variety $Q^{(1)}$ is composed of all $\left(p ; \omega_{0}, \omega_{1}, \omega_{2}, \omega_{3}\right) \in J^{3}(M, m)$ satisfying $\left(p ; \omega_{0}\right.$, $\left.\omega_{1}, \omega_{2}\right) \in Q$ and

$$
\begin{gathered}
\left\langle\omega_{3}(u, z, x), \omega_{1}(y)\right\rangle+\left\langle\omega_{2}(z, x), \omega_{2}(u, y)\right\rangle=0, \\
\left\langle\omega_{3}(v, z, x), \omega_{2}(u, y)\right\rangle+\left\langle\omega_{2}(z, x), \omega_{3}(v, u, y)\right\rangle-\left\langle\omega_{3}(v, u, x), \omega_{2}(z, y)\right\rangle \\
-\left\langle\omega_{2}(u, x), \omega_{3}(v, z, y)\right\rangle=-g\left(\nabla_{v} R(z, u) x, y\right) \quad \text { for any } x, y, z, u, v \in T_{p} .
\end{gathered}
$$

In the subsequent sections we shall mainly concerned with the variety $Q$. In connection with the variety $Q$ we make some definitions.

Let $\beta=\left(p ; \omega_{0}, \omega_{1}, \omega_{2}\right) \in Q$. By definition the symbol $\mathfrak{q}_{\beta}$ of the variety $Q$ at $\beta$ is the subspace of $S^{2} T_{p}^{*} \otimes N_{\alpha}$ consisting of all $\xi \in S^{2} T_{p}^{*} \otimes N_{\alpha}$ such that

$$
\begin{array}{cc}
\left\langle\xi(z, x), \omega_{2}(u, y)\right\rangle+\left\langle\omega_{2}(z, x), \xi(u, y)\right\rangle & -\left\langle\xi(u, x), \omega_{2}(z, y)\right\rangle \\
-\left\langle\omega_{2}(u, x), \xi(z, y)\right\rangle=0 \quad \text { for any } x, y, z, u \in T_{p},
\end{array}
$$

where we set $\alpha=\pi_{1}^{2}(\beta)$. We also denote by $\mathfrak{q}_{\beta}^{(1)}$ the first prolongation of the symbol $\mathfrak{q}_{\beta}$, i.e., $\mathfrak{q}_{\beta}^{(1)}=T^{*} \otimes \mathfrak{q}_{\beta} \cap S^{3} T_{p}^{*} \otimes N_{\alpha}$.

\section{§ 2. Formal Gaussian varieties}

Let $T$ be a finite dimensional real vector space and $T^{*}$ be the dual vector space of $T . \quad$ By $\otimes^{k} T^{*}\left(\right.$ resp. $\left.S^{k} T^{*}\right)$ we mean the vector space of covariant $k$-tensors (resp. symmetric $k$-tensors) of $\boldsymbol{T}$.

By definition an element $C \in \otimes^{4} T^{*}$ is called curvature like if it satisfies the following:

$$
\begin{aligned}
& C(x, y, z, w)=-C(y, x, z, w)=-C(x, y, w, z), \\
& C(x, y, z, w)+C(y, z, x, w)+C(z, x, y, w)=0 \quad \text { for } x, y, z, w \in T .
\end{aligned}
$$

We denote by $K(T)$ the vector space of all curvature like tensors. 
Let $N$ be another finite dimensional real vector space with an inner product $\langle$,$\rangle .$ For each $\alpha \in S^{2} T^{*} \otimes N$, we denote by $\Omega(\alpha)$ the element in $K(T)$ defined by

$$
\Omega(\alpha)(x, y, z, w)=\langle\alpha(x, z), \alpha(y, w)\rangle-\langle\alpha(x, w), \alpha(y, z)\rangle \quad \text { for } x, y, z, w \in T .
$$

Let $C$ be any element in $K(T)$. By $\mathscr{G}(C)$ we mean the inverse image of $C$ by the map $S^{2} T^{*} \otimes N \ni \alpha \rightarrow \Omega(\alpha) \in K(T) . \quad \mathscr{G}(C)$ is called the formal Gaussian variety with respect to $C$.

Let $\alpha \in \mathscr{G}(C)$. Define a linear map $\Omega_{* \alpha}: S^{2} T^{*} \otimes N \rightarrow K(T)$ by setting

$$
\begin{aligned}
\Omega_{* \alpha}(\beta)(x, y, z, w)= & \langle\beta(x, z), \alpha(y, w)\rangle+\langle\alpha(x, z), \beta(y, w)\rangle \\
& -\langle\beta(x, w), \alpha(y, z)\rangle-\langle\alpha(x, w), \beta(y, z)\rangle \\
& \text { for } \beta \in S^{2} T^{*} \otimes N, x, y, z, w \in T .
\end{aligned}
$$

We denote by $\mathrm{g}_{\alpha}$ the kernel of the map $\Omega_{a}$, which may be identified with the tangent space to the variety $\mathscr{G}(\Omega(\alpha))$ at $\alpha$. We also denote by $\mathrm{g}_{\alpha}^{(1)}$ the first prolongation of the subspace $\mathfrak{g}_{\alpha}$ of $S^{2} T^{*} \otimes N$, i.e., $\mathfrak{g}_{\alpha}^{(1)}=T^{*} \otimes \mathfrak{g}_{\alpha} \cap S^{3} T^{*} \otimes N$.

Let $\langle$,$\rangle be an inner product of T$. For each $k \in R$ let us define $C_{k} \in K(T)$ by

$$
C_{k}(x, y, z, w)=k(\langle x, z\rangle\langle y, w\rangle-\langle x, w\rangle\langle y, z\rangle) \quad \text { for } x, y, z, w \in T .
$$

We say that $C_{k}$ is the curvature like tensor of constant curvature with sectional curvature $k$.

Let $O(T)$ (resp. $O(N)$ ) be the orthogonal group of $T$ (resp. $N$ ) with respect to the given inner product $\langle$,$\rangle of T$ (resp. $N$ ) and $\mathfrak{o}(T)$ (resp. $\mathfrak{o}(N))$ be the Lie algebra of $O(T)$ (resp. $O(N))$.

Let $a \in O(N), t \in O(T), \chi \in S^{l} T^{*} \otimes N$ and $C \in K(T)$. Let us define $a \chi^{t} \in S^{l} T^{*} \otimes N$ and $C^{t} \in K(T)$ by setting

$$
\begin{aligned}
& a \chi^{t}\left(x_{1}, \cdots, x_{l}\right)=a\left(\chi\left(t x_{1}, \cdots, t x_{l}\right)\right) \\
& C^{t}\left(x_{1}, x_{2}, x_{3}, x_{4}\right)=C\left(t x_{1}, t x_{2}, t x_{3}, t x_{4}\right) \quad \text { for } x_{1}, \cdots, x_{4}, \cdots, x_{l} \in T .
\end{aligned}
$$

It is straightforward to see that if $\alpha \in \mathscr{G}(C)$, then $a \alpha^{t} \in \mathscr{G}\left(C^{t}\right)$. Similarly if $\beta \in \mathfrak{g}_{\alpha}$ (resp. $\gamma \in \mathfrak{g}_{\alpha}^{(1)}$ ), then $a \beta^{t} \in \mathfrak{g}_{a \alpha^{t}}$ (resp. $\left.\alpha \gamma^{t} \in \mathfrak{g}_{a \alpha}^{(1)}\right)$.

Let us consider the case $C=C_{k}$. Since $C_{k}^{t}=C_{k}$ holds for any $t \in O(T)$, we have

Proposition 2.1. The formal Gaussian variety $\mathscr{G}\left(C_{k}\right)$ is invariant under the action of the product group $O(N) \times O(T)$ on $S^{2} T^{*} \otimes N$ defined by

$$
O(N) \times O(T) \times S^{2} T^{*} \otimes N \ni((a, t), \alpha) \rightarrow a \alpha^{t} \in S^{2} T^{*} \otimes N .
$$

Proposition 2.1 is useful in the consideration of the formal Gaussian variety $\mathscr{G}\left(C_{k}\right)$.

\section{§3. The formal Gaussian variety $\mathscr{G}\left(C_{k}\right)$ with $k<0$}

In this and the next sections we shall investigate the formal Gaussian variety 
$\mathscr{G}\left(C_{k}\right)$ with $k<0$. Our main aim is to show

Theorem 3.1. Assume that $\operatorname{dim} N=\operatorname{dim} T-1=n$ and $k<0$. Then there exists an open set $O$ in $S^{2} T^{*} \otimes N$ such that:

(1) $\mathscr{G}\left(C_{k}\right) \cap O$ is an $n(n+1)$-dimensional submanifold of $S^{2} T^{*} \otimes N$.

(2) For each $\alpha \in \mathscr{G}\left(C_{k}\right) \cap O$, there exists a vector $e \in T$ such that

$$
e\lrcorner \beta \neq 0 \quad \text { for any } \beta \in \mathfrak{g}_{\alpha}, \beta \neq 0 \text {. }
$$

(3) $\operatorname{dim} \mathfrak{g}_{\alpha}^{(1)}=n(n+1)$ for any $\alpha \in \mathscr{G}\left(C_{k}\right) \cap O$.

Remark. In order to prove the theorem we have only to prove it in the case where $k=-1$. In fact consider the linear endomorphism of $S^{2} T^{*} \otimes N$ given by $S^{2} T^{*} \otimes N \ni \alpha \rightarrow \sqrt{-k} \alpha \in S^{2} T^{*} \otimes N$. It is clear that this endomorphism maps $\mathscr{G}\left(C_{-1}\right)$ onto $\mathscr{G}\left(C_{k}\right)$. Moreover we have $\mathfrak{g}_{\alpha}=\mathfrak{g}_{\sqrt{ }-k \alpha}$ and hence $\mathfrak{g}_{\alpha}^{(1)}=\mathfrak{g}_{\sqrt{-k} \alpha}^{(1)}$.

In the following we shall simply write $\mathscr{G}$ instead of $\mathscr{G}\left(C_{-1}\right)$.

Let $\left\{e_{a}\right\}_{0 \leqq a \leqq n}$ (resp. $\left\{v_{i}\right\}_{1 \leqq i \leqq n}$ ) be an orthonormal basis of $\boldsymbol{T}$ (resp. $N$ ). Making use of these basis, we shall express $N$-valued covariant tensors of $T$ in terms of their coefficients. Let $\chi \in \otimes^{\imath} T^{*} \otimes N$. Define an element $X=\left(X_{a_{1}, \cdots, a_{l}}^{k}\right)_{\substack{0 \leqq a_{1}, \ldots, a_{l} \leqq n \\ 1 \leqq k \leqq n}} \in R^{n(n+1)^{l}}$ by

$$
X_{a_{1}, \cdots, a_{l}}^{k}=\left\langle\chi\left(e_{a_{1}}, \cdots, e_{a_{l}}\right), v_{k}\right\rangle \quad \text { for } \quad 0 \leqq a_{1}, \cdots, a_{l} \leqq n, 1 \leqq k \leqq n .
$$

Let $\alpha \in \mathscr{G}$ and let $A=\left(A_{a b}^{k}\right)_{\substack{0 \leq a, b \leq n \\ 1 \leq k \leq n}} \in R^{n(n+1)^{2}}$ be the coefficients of $\alpha$. Then we have

$$
\begin{gathered}
A_{a b}{ }^{k}=A_{b a}{ }^{k}, \\
\sum_{p=1}^{n}\left(A_{a c}{ }^{p} A_{b d}{ }^{p}-A_{a d}{ }^{p} A_{b c}{ }^{p}\right)= \\
\text { for } 0 \leqq a, b, c, d \leqq n, 1 \leqq k \leqq n,
\end{gathered}
$$

where $\delta$ means the Kronecker's delta. Conversely, it is clear that any $\alpha \in \bigotimes^{2} T^{*} \otimes N$ whose coefficients $A=\left(A_{a b}{ }^{k}\right)_{\substack{0 \leqq n, b \leq n \\ 1 \leqq k \leqq n}}$ satisfy (3.1) and (3.2) is contained in $\mathscr{G}$.

Let $\alpha \in \mathscr{G}$. Assume that $\alpha$ satisfies the following

$$
\alpha\left(e_{0}, e_{0}\right)=0, \quad \alpha\left(e_{0}, e_{i}\right)=v_{i} \quad \text { for } \quad 1 \leqq i \leqq n .
$$

Then we have $A_{00}{ }^{k}=0, A_{0 i}{ }^{k}=\delta_{i k}$ for $1 \leqq i, k \leqq n$. Hence by (3.1) and (3.2) we obtain

$$
\begin{gathered}
A_{i j}{ }^{k}=A_{j i}{ }^{k}=A_{i k}{ }^{j}, \\
\sum_{p=1}^{n}\left(A_{i k}{ }^{p} A_{j l p}-A_{i l}{ }^{p} A_{j k p}\right)=-\left(\delta_{i k} \delta_{\jmath l}-\delta_{i l} \delta_{j k}\right) \\
\text { for } 1 \leqq i, j, k, l \leqq n .
\end{gathered}
$$

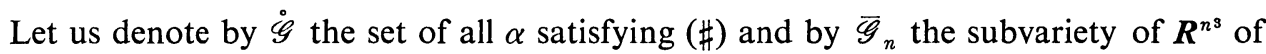
all $A=\left(A_{i j}{ }^{k}\right)_{1 \leqq i, j, k \leqq n} \in \boldsymbol{R}^{n 3}$ satisfying the system of equations (3.3) and (3.4). Clearly we can identify $\mathscr{G}$ and $\overline{\mathscr{G}}_{n}$ in a natural way. 
Let $A=\left(A_{i j}{ }^{k}\right)_{1 \leqq i, j, k \leqq n} \in \overline{\mathscr{G}}_{n}$. By $\left(\overline{\mathfrak{g}}_{n}\right)_{A}$, 'we mean the vector space of all $B=$ $\left(B_{i j}{ }^{k}\right)_{1 \leqq i, j, k \leqq n} \in \boldsymbol{R}^{n^{3}}$ such that

$$
\begin{gathered}
B_{i j}{ }^{k}=B_{j i}{ }^{k}=B_{i k}{ }^{j}, \\
\sum_{p=1}^{n}\left({B_{i k}}^{p} A_{j l}{ }^{p}+A_{i k}{ }^{p}{B_{j l}}^{p}-B_{i l}{ }^{p} A_{j k}{ }^{p}-A_{i l}^{p} B_{j k}{ }^{p}\right)=0 \\
\text { for } 1 \leqq i, j, k, l \leqq n .
\end{gathered}
$$

Naturally $\left(\bar{g}_{n}\right)_{A}$ may be idetified with the tangent space to the subvariety $\overline{\mathscr{G}}_{n}$ at $A$. We also denote by $\left(\overline{\mathrm{g}}_{n}^{(1)}\right)_{A}$ the vector space of all $C=\left({ }^{l} C_{i j}{ }^{k}\right)_{1 \leqq i, j, k, l \leqq n} \in \boldsymbol{R}^{n^{4}}$ such that

$$
\begin{gathered}
{ }^{l} C_{i j}{ }^{k}={ }^{i} C_{l j}{ }^{k} \\
{ }^{l} C_{i j}{ }^{k}={ }^{l} C_{j l}{ }^{k}={ }^{l} C_{i k}{ }^{j}, \\
\sum_{p=1}^{n}\left({ }^{q} C_{i k}{ }^{p} A_{j l}{ }^{p}+A_{i k}{ }^{p q} C_{j l}{ }^{p}-{ }^{q} C_{i l}{ }^{p} A_{j k}{ }^{p}-A_{i l}{ }^{p q} C_{j k}{ }^{p}\right)=0 \\
\text { for } 1 \leqq i, j, k, l \leqq n .
\end{gathered}
$$

We prove

Proposition 3.2. There exists an open set $U$ of $\boldsymbol{R}^{n s}$ such that:

(1) $\overline{\mathscr{G}}_{n} \cap U$ is a $\frac{1}{2} n(n+1)$-dimensional submanifold of $\boldsymbol{R}^{n s}$.

(2) For each $A \in \overline{\mathscr{G}}_{n} \cap U$, there exists a vector $x=\left(x_{1}, \cdots, x_{n}\right) \in \boldsymbol{R}^{n}$ having the following property:

If $B=\left(B_{i j}{ }^{k}\right)_{1 \leqq i, j, k \leqq n} \in\left(\overline{\mathrm{g}}_{n}\right)_{A}$ and $B \neq 0$, then

$$
\sum_{p=1}^{n} x_{k} B_{i j}{ }^{k} \neq 0 \quad \text { for some }(i, j)(1 \leqq i, j \leqq n) .
$$

(3) $\operatorname{dim}\left(\overline{\mathfrak{g}}_{n}^{(1)}\right)_{A}=\frac{1}{2} n(n+1)$ for any $A \in \overline{\mathscr{G}}_{n} \cap U$.

Before proceeding to the proof of Proposition 3.2, we first note the following. Let $a=\left(a_{i}{ }^{j}\right)_{1 \leqq i, j \leqq n}$ be an orthogonal matrix, i.e., $\sum_{k=1}^{n} a_{k}{ }^{i} a_{k}{ }^{j}=\delta_{i j}$ and let $X=$ $\left(X_{i j}{ }^{k}\right)_{1 \leqq i, j, k \leqq n} \in \boldsymbol{R}^{n^{8}}$ and $Y=\left({ }^{l} Y_{i j}{ }^{k}\right)_{1 \leqq i, j, k, l \leqq n} \in \boldsymbol{R}^{n 4}$. Define $X^{a}=\left(\left(X^{a}\right)_{i j}{ }^{k}\right)_{1 \leqq i, j, k \leqq n} \in \boldsymbol{R}^{n^{3}}$ and $Y^{a}=\left({ }^{l}\left(Y^{a}\right)_{i j}{ }^{k}\right)_{1 \leqq i, j, k, l \leqq n} \in \boldsymbol{R}^{n^{4}}$ by setting

$$
\begin{aligned}
\left(X^{a}\right)_{i j}{ }^{k} & =\sum_{p, q, r=1}^{n} a_{i}{ }^{p} a_{j}{ }^{q} a_{k}{ }^{r} X_{p q}{ }^{r}, \\
{ }^{l}\left(Y^{a}\right)_{i j}{ }^{k} & =\sum_{p, q, r, s=1}^{n} a_{l}{ }^{s} a_{i}{ }^{p} a_{j}^{q} a_{k}{ }^{r s} Y_{p q}{ }^{r} \quad \text { for } \quad 1 \leqq i, j, k, l \leqq n .
\end{aligned}
$$

By simple calculations we know that if $A \in \overline{\mathscr{G}}_{n}$ then $A^{a} \in \overline{\mathscr{G}}_{n}$ and that if $B \in\left(\overline{\mathfrak{g}}_{n}\right)_{A}$ (resp. $\left.C \in\left(\overline{\mathfrak{g}}_{n}^{(1)}\right)_{A}\right)$, then $B^{a} \in\left(\overline{\mathfrak{g}}_{n}\right)_{A^{a}}$ (resp. $\left.C^{a} \in\left(\overline{\mathfrak{g}}_{n}^{(1)}\right)_{A^{a}}\right)$.

From now on let us assume that $n \geqq 2$ and $\overline{\mathscr{G}}_{n-1} \neq \phi$. Let $\lambda \in \boldsymbol{R}_{*}=\boldsymbol{R}-\{0\}$ and let $\hat{A}=\left(\hat{A}_{i j}{ }^{k}\right) \in \overline{\mathscr{G}}_{n-1}$. Define an element $\varphi(\lambda, \hat{A})=\left(\varphi(\lambda, \hat{A})_{i j}{ }^{k}\right)_{1 \leqq i, j, k \leqq n} \in \boldsymbol{R}^{n^{3}}$ by

$$
\varphi(\lambda, \hat{A})_{i j}{ }^{k}=\sqrt{1+\lambda^{2}} \hat{A}_{i j}{ }^{k},
$$


$(*)$

$$
\begin{gathered}
\varphi(\lambda, \hat{A})_{i j}{ }^{n}=\varphi(\lambda, \hat{A})_{i n}{ }^{j}=\varphi(\lambda, \hat{A})_{n i}{ }^{j}=\lambda \delta_{i j}, \\
\varphi(\lambda, \hat{A})_{n n}{ }^{i}=\varphi(\lambda, \hat{A})_{n i}{ }^{n}=\varphi(\lambda, \hat{A})_{i n}{ }^{n}=0, \quad \varphi(\lambda, \hat{A})_{n n}{ }^{n}=\lambda-1 / \lambda \\
\quad \text { for } 1 \leqq i, j, k \leqq n-1 .
\end{gathered}
$$

It is easily observed that $\varphi(\lambda, \hat{A}) \in \overline{\mathscr{G}}_{n}$. We now define a map $\Phi: \boldsymbol{R}_{*} \times \overline{\mathscr{G}}_{n-1} \times \boldsymbol{R}^{n-1}$ $\rightarrow \boldsymbol{R}^{n 3}$ by

$$
\Phi(\lambda, \hat{A}, \xi)=\varphi(\lambda, \hat{A})^{\exp \xi} \quad \text { for }(\lambda, \hat{A}, \xi) \in \boldsymbol{R}_{*} \times \overline{\mathscr{G}}_{n-1} \times \boldsymbol{R}^{n-1},
$$

where, for $\xi=\left(\xi_{1}, \cdots, \xi_{n-1}\right) \in \boldsymbol{R}^{n-1}$, we mean by $\bar{\xi}$ the skew symmetric matrix of the form:

$$
\tilde{\xi}=\left(\begin{array}{cc}
0 & -\xi_{1} \\
\vdots & -\xi_{n-1} \\
\xi_{1} \cdots \xi_{n-1} & 0
\end{array}\right) .
$$

Since $\varphi(\lambda, \hat{A}) \in \overline{\mathscr{G}}_{n}$, we have $\Phi(\lambda, \hat{A}, \xi) \in \overline{\mathscr{G}}_{n}$. Let $(\lambda, \hat{A}) \in \boldsymbol{R}_{*} \times \overline{\mathscr{G}}_{n-1}$. We denote by $\Phi_{*}=\Phi_{*(\lambda, \hat{A}, 0)}$ the differential of the map $\Phi$ at $(\lambda, \hat{A}, 0)$, where 0 means the zero vector $(0, \cdots, 0)$ in $\boldsymbol{R}^{n-1}$. In a natural way, the tangent space the variety $\boldsymbol{R}_{*} \times \overline{\mathscr{G}}_{n-1} \times \boldsymbol{R}^{n-1}$ at $(\lambda, \hat{A}, 0)$ may be identified with the direct sum $\boldsymbol{R} \oplus\left(\overline{\mathrm{g}}_{n-1}\right)_{\hat{\boldsymbol{A}}} \oplus \boldsymbol{R}^{n-1}$. Hence $\Phi_{*}$ induces a linear map of $\boldsymbol{R} \oplus\left(\overline{\mathrm{g}}_{n-1}\right)_{\hat{A}} \oplus \boldsymbol{R}^{n-1}$ into $\boldsymbol{R}^{n 3}$. We also denote it by $\Phi_{*}$.

Let $\mu \in \boldsymbol{R}, \hat{B}=\left(\hat{B}_{i j}{ }^{k}\right)_{1 \leq i, j, k \leq n-1} \in\left(\overline{\mathfrak{g}}_{n-1}\right)_{\hat{A}}$ and $\xi=\left(\xi_{1}, \cdots, \xi_{n-1}\right) \in \boldsymbol{R}^{n-1}$. We note that the vectors $\Phi_{*}(\mu), \Phi_{*}(B)$ and $\Phi_{*}(\xi)$ in $\boldsymbol{R}^{n 3}$ are necessarily contained in $\left(\overline{\mathfrak{g}}_{n}\right)_{\varphi(\lambda, \hat{A})}$. By using the coefficients they are explicitly represented as follows:

$$
\begin{aligned}
& \Phi_{*}(\mu)_{i j}{ }^{k}=\left(\lambda / \sqrt{1+\lambda^{2}}\right) \mu \hat{A}_{i j}{ }^{k}, \Phi_{*}(\mu)_{i j}{ }^{n}=\mu \delta_{i j}, \\
& \Phi_{*}(\mu)_{i n}{ }^{n}=0, \Phi_{*}(\mu)_{n n}{ }^{n}=\left(1 / \lambda^{2}\right)\left(1+\lambda^{2}\right) \mu ; \\
& \Phi_{*}(\hat{B})_{i j}{ }^{k}=\sqrt{1+\lambda^{2} \hat{B}_{i j}{ }^{k}} \\
& \Phi_{*}(\hat{B})_{i j}{ }^{n}=\Phi_{*}(\hat{B})_{i n}{ }^{n}=\Phi_{*}(\hat{B})_{n n}{ }^{n}=0 \\
& \Phi_{*}(\xi)_{i j}{ }^{k}=\lambda\left(\xi_{i} \delta_{j k}+\xi_{j} \delta_{k i}+\xi_{k} \delta_{i j}\right), \\
& \Phi_{*}(\xi)_{i j}{ }^{n}=-\sqrt{1+\lambda^{2}}\left(\sum_{p=1}^{n} \xi_{p} \hat{A}_{i j}{ }^{p}\right), \\
& \Phi_{*}(\xi)_{i n}{ }^{n}=-(1 / \lambda)\left(1+\lambda^{2}\right) \xi_{i}, \Phi_{*}(\xi)_{n n}{ }^{n}=0 \quad \text { for } \quad 1 \leqq i, j, k \leqq n-1 .
\end{aligned}
$$

Lemma 3.3. For each $B \in\left(\overline{\mathfrak{g}}_{n}\right)_{\varphi(\lambda, \hat{\mathrm{A}})}$, there exists a unique $(\mu, \hat{B}, \xi) \in \boldsymbol{R} \oplus\left(\overline{\mathfrak{g}}_{n-1}\right)_{\hat{A}}$ $\oplus \boldsymbol{R}^{n-1}$ such that

$$
B=\Phi_{*}(\mu)+\Phi_{*}(\hat{B})+\Phi_{*}(\xi) .
$$

Proof. We first suppose that $\Phi_{*}(\mu)+\Phi_{*}(\hat{B})+\Phi_{*}(\xi)=0$ for some $(\mu, \hat{B}, \xi) \in \boldsymbol{R}$ $\oplus\left(\overline{\mathrm{g}}_{n-1}\right)_{\hat{A}} \oplus \boldsymbol{R}^{n+1}$. Then by $(* *)$ we can easily obtain $\mu=\hat{B}=\xi=0$. This implies the uniqueness. Next we show the decomposition. Let $B=\left(B_{i j}{ }^{k}\right)_{1 \leq i, j, k \leqq n} \in\left(\overline{\mathrm{g}}_{n}\right)_{\varphi(\lambda, \hat{\mathrm{A}})}$. Take $\mu \in \boldsymbol{R}$ and $\xi=\left(\xi_{1}, \cdots, \xi_{n-1}\right) \in \boldsymbol{R}^{n-1}$ so that $B_{n n}{ }^{n}=\left(1 / \lambda^{2}\right)\left(1+\lambda^{2}\right) \mu, B_{i n}{ }^{n}=-(1 / \lambda)\left(1+\lambda^{2}\right) \xi_{i}$ for $1 \leqq i \leqq n$. We set $\bar{B}=B-\left(\Phi_{*}(\mu)+\Phi_{*}(\xi)\right)$. Then $\bar{B} \in\left(\overline{\mathrm{g}}_{n}\right)_{\varphi(\lambda, \hat{A})}, \bar{B}_{n n}{ }^{n}=0$ and $\bar{B}_{i n}{ }^{n}$ 
$=0$ for $1 \leqq i \leqq n$. Substituting $B=\bar{B}$ into (3.6), we have $\bar{B}_{i j}{ }^{n}=0$ for $1 \leqq i, j \leqq n-1$ and

$$
\begin{array}{r}
\sum_{p=1}^{n}\left(\bar{B}_{i k}{ }^{p} \hat{A}_{j l}{ }^{p}+\hat{A}_{i k}{ }^{p} \bar{B}_{j l}{ }^{p}-\bar{B}_{i l}{ }^{p} \hat{A}_{j k}{ }^{p}-\hat{A}_{i l}{ }^{p} \bar{B}_{j k}{ }^{p}\right)=0 \\
\text { for } 1 \leqq i, j, k, l \leqq n-1 .
\end{array}
$$

Thus if we set $\hat{B}_{i j}{ }^{k}=\left(1 / \sqrt{1+\lambda^{2}}\right) \bar{B}_{i j}{ }^{k}$ for $1 \leqq i, j, k \leqq n-1$, then $\hat{B}=\left(\hat{B}_{i j}{ }^{k}\right)_{1 \leqq i, j, k \leqq n-1} \epsilon$ $\left(\overline{\mathfrak{g}}_{n-1}\right)_{\hat{A}}$ and $\bar{B}=\Phi_{*}(\hat{B})$. Hence we have $B=\Phi_{*}(\mu)+\Phi_{*}(\hat{B})+\Phi_{*}(\xi)$ Q.E.D.

Proof of Proposition 3.2. We proceed by induction on $n$. It is easy to see that the proposition holds for $n=1$. We now assume that the proposition holds $n-1$ with $n \geqq 2$. Then there exists an open set $\hat{U}$ in $\boldsymbol{R}^{(n-1)^{3}}$ such that:

(1') $\overline{\mathscr{G}}_{n-1} \cap \hat{U}$ is a $\frac{1}{2} n(n-1)$-dimensional submanifold of $\boldsymbol{R}^{(n-1)^{3}}$.

(2') For each $\hat{A} \in \overline{\mathscr{G}}_{n-1} \cap \hat{U}$, there exists a vector $x=\left(x_{1}, \cdots, x_{n-1}\right) \in \boldsymbol{R}^{n-1}$ having the following property:

If $\hat{B}=\left(\hat{B}_{i j}{ }^{k}\right)_{1 \leqq i, j, k \leqq n-1} \in\left(\overline{\mathfrak{g}}_{n-1}\right)_{\hat{A}}$ and $B \neq 0$, then

$$
\sum_{p=1}^{n-1} x_{k} B_{i j}{ }^{k} \neq 0 \quad \text { for some }(i, j)(1 \leqq i, j \leqq n-1) .
$$

(3') $\operatorname{dim}\left(\overline{\mathfrak{g}}_{n-1}\right)_{\hat{A}}=\frac{1}{2} n(n-1)$ for any $\hat{A} \in \overline{\mathscr{G}}_{n-1} \cap \hat{U}$.

Let us take any $\lambda_{0} \in \boldsymbol{R}_{*}, \hat{A}_{0} \in \overline{\mathscr{G}}_{n-1} \cap \hat{U}$ and a sufficiently small open set $\tilde{U}$ in $\boldsymbol{R}_{*}$ $\times\left(\overline{\mathscr{G}}_{n-1} \cap \hat{U}\right) \times \boldsymbol{R}^{n-1}$ containing $\left(\lambda_{0}, \hat{A}_{0}, 0\right)$. In view of Lemma 3.3 , we know that the differential $\Phi_{*}$ of at $\left(\lambda_{0}, \hat{A}_{0}, 0\right)$ is injective. Hence we may assume that the restriction $\Phi_{1 \tilde{U}}: \widetilde{U} \rightarrow \boldsymbol{R}^{n^{3}}$ of the map $\Phi$ to $\tilde{U}$ is an imbedding. Because of the assumption $\left(1^{\prime}\right)$, the image $\Phi(\tilde{U})$ of $\tilde{U}$ forms $a \frac{1}{2} n(n-1)$-dimensional submanifold of $\boldsymbol{R}^{n s}$. Moreover we know $\left(\overline{\mathfrak{g}}_{n}\right)_{\varphi\left(\lambda_{0}, \hat{A}_{0}\right)}=T_{\varphi\left(\lambda_{0}, \hat{A}_{0}\right)} \Phi(\tilde{U})$. We now notice the following

Lemma 3.4. Let $f_{1}, \cdots, f_{r}$ be differentiable functions on a manifold $M$ and let $\mathscr{V}$ be the subvariety of $M$ defined by the system of equations $f_{1}=\cdots=f_{r}=0$. Assume that there is a submanifold $S$ of $M$ and a point $p \in S$ such that

(i) $S \subset \mathscr{V}$;

(ii) $T_{p}(S)=\left\{x \in T_{p}(M) \mid d f_{1}(x)=\cdots=d f_{r}(x)=0\right\}$.

Then there exists a neighborhood $U$ of $p$ in $M$ such that $\mathscr{V} \cap U=S \cap U$.

The proof of the lemma is left to the readers.

By virtue of Lemma 3.4, we know that there exists an open neighborhood $U$ of $\varphi\left(\lambda_{0}, \hat{A_{0}}\right)$ in $R^{n 3}$ such that $\overline{\mathscr{G}}_{n} \cap U=\Phi(\tilde{U}) \cap U$. This implies (1) of the proposition.

Next we show (2). Let $A \in \overline{\mathscr{G}}_{n} \cap U$. Since $A \in \Phi(\hat{U})$, we may write $A=\varphi(\lambda, \hat{A})^{\exp \xi}$ by using a suitable $(\lambda, \hat{A}, \xi) \in \tilde{U}$. Hence, in order to show (2), it suffices to deal with the case $A=\varphi(\lambda, \hat{A})$. Let $\hat{x}=\left(\hat{x}_{1}, \cdots, \hat{x}_{n-1}\right) \in \boldsymbol{R}^{n-1}$ be the vector stated in $\left(2^{\prime}\right)$. We may assume that $\left|\hat{x}_{i}\right| \ll 1$ for $1 \leqq i \leqq n-1$. We define $x=\left(x_{1}, \cdots, x_{n}\right) \in \boldsymbol{R}^{n}$ by $x_{i}=\hat{x}_{i}$ for $1 \leqq i \leqq n-1$ and $x_{n}=1$. Let us show the vector $x$ defined above has the property stated in (2). Let $B=\left(B_{p q}{ }^{r}\right)_{1 \leqq p, q, r \leqq n} \in\left(\overline{\mathfrak{g}}_{n}\right)_{\varphi(\lambda, \hat{A})}$ and suppose $\sum_{r=1}^{n} x_{r} B_{p q}{ }^{r}=0$ for any $(p, q)(1 \leqq p, q \leqq n)$. By using the decomposition $B=\Phi_{*}(\mu)+\Phi_{*}(\hat{B})+\Phi_{*}(\xi)$ in 
Lemma 3.3 and the formulas $(* *)$ for $\Phi_{*}(\mu), \Phi_{*}(\hat{B}), \Phi_{*}(\xi)$, we have

$$
\begin{aligned}
& \sum_{k=1}^{n-1}\left\{\lambda\left(\hat{x}_{j} \delta_{i k}+\hat{x}_{i} \delta_{j k}+\hat{x}_{k} \delta_{i j}\right)-\sqrt{1+\lambda^{2}} \hat{A}_{i j}{ }^{k}\right\} \xi_{k} \\
& +\left(\delta_{i j}+\frac{\lambda}{\sqrt{1+\lambda^{2}}} \sum_{k=1}^{n-1} \hat{x}_{k} \hat{A}_{i j}{ }^{k}\right) \mu+\sqrt{1+\lambda^{2}}\left(\sum_{k=1}^{n-1} \hat{x}_{k} \hat{B}_{i j}{ }^{k}\right)=0 \\
& \text { for } 1 \leqq i, j \leqq n-1 ; \\
& -\frac{1}{\lambda}\left(1+\lambda^{2}\right) \sum_{j=1}^{n-1}\left(\delta_{i j}+\frac{\lambda}{\sqrt{1+\lambda^{2}}} \sum_{j=1}^{n-1} \hat{x}_{k} \hat{A}_{i j}{ }^{k}\right) \xi_{j}+\hat{x}_{i} \mu=0 \\
& \text { for } 1 \leqq i \leqq n-1 ; \\
& \mu-\lambda \sum_{k=1}^{n-1} \hat{x}_{k} \xi_{k}=0 .
\end{aligned}
$$

Since $\left|\hat{x}_{i}\right| \ll 1$, it follows from (3.11) and (3.12) that $\mu=\xi_{1}=\cdots=\xi_{n-1}=0$. Hence by (3.10) we have $\sum_{k=1}^{n} \hat{x}_{k} \hat{B}_{i j}{ }^{k}=0$ for any $1 \leqq i, j \leqq n-1$. Therefore by the assumption (2'), we obtain $\hat{B}=0$. This completes the proof of (2).

Finally we show (3) of the proposition. As in the proof of (2), we may assume $A=\varphi(\lambda, \hat{A})$. Let $C=\left({ }^{s} C_{p q}{ }^{r}\right)_{1 \leqq p, q, r, s \leqq n} \in\left(\overline{\mathfrak{g}}_{n}^{(1)}\right)_{\varphi(\lambda, \hat{A})}$. For each $s(1 \leqq s \leqq n)$, we denote by ${ }^{s} C$ the element of $R^{n 3}$ given by ${ }^{s} C=\left({ }^{8} C_{p q}{ }^{r}\right)_{1 \leqq p, q, r \leqq n}$. Clearly we have ${ }^{s} C \in\left(\overline{\mathrm{g}}_{n}\right)_{\varphi(\lambda, \hat{\mathrm{A}})}$ for $1 \leqq s \leqq n$. By Lemma 3.3, there exist ${ }^{s} \mu \in R,{ }^{s} \hat{B} \in\left(\overline{\mathrm{g}}_{n-1}\right)_{\hat{A}}$ and ${ }^{s} \xi \in \boldsymbol{R}^{n-1}$ such that

$$
{ }^{s} C=\Phi_{*}\left({ }^{s} \mu\right)+\Phi_{*}\left({ }^{s} \hat{B}\right)+\Phi_{*}\left({ }^{s} \xi\right) .
$$

Since ${ }^{s} C_{p q}{ }^{r}={ }^{p} C_{s q}{ }^{r}$, we have

$$
{ }^{i} \mu=-\lambda^{n} \xi_{i} \quad \text { for } \quad 1 \leqq i \leqq n-1 ;
$$

$$
\begin{aligned}
& { }^{j} \xi_{i}=\frac{\lambda}{\sqrt{1+\lambda^{2}}}\left(\sum_{l=1}^{n-1}{ }^{n} \xi_{l} \hat{A}_{i j}{ }^{l}-\frac{1}{\sqrt{1+\lambda^{2}}}{ }^{n} \mu \delta_{i j}\right) \quad \text { for } \quad 1 \leqq i, j \leqq n-1 \text {; } \\
& { }^{n} \hat{B}_{i j}{ }^{k}=-\frac{\lambda}{\sqrt{1+\lambda^{2}}}\left(2^{n} \xi_{i} \delta_{j k}+{ }^{n} \xi_{j} \delta_{i k}+{ }^{n} \xi_{k} \delta_{i j}\right)-\frac{\lambda}{\sqrt{1+\lambda^{2}}}\left(\sum_{p, q=1}^{n-1}{ }^{n} \xi_{q} \hat{A}_{i q}{ }^{p} \hat{A}_{j k}{ }^{p}\right) \\
& \text { for } 1 \leqq i, j, k \leqq n-1 \text {; } \\
& { }^{l} \hat{B}_{i j}{ }^{k}-{ }^{i} \hat{B}_{l j}{ }^{k}=\frac{\lambda}{\sqrt{1+\lambda^{2}}}\left({ }^{i} \xi_{j} \delta_{l k}+{ }^{i} \xi_{k} \delta_{l j}-{ }^{l} \xi_{j} \delta_{i k}-{ }^{l} \xi_{k} \delta_{i j}\right) \\
& +\frac{\lambda}{1+\lambda^{2}}\left({ }^{i} \mu \hat{A}_{l j}{ }^{k}-{ }^{l} \mu \hat{A}_{i j}{ }^{k}\right) \quad \text { for } \quad 1 \leqq i, j, k, l \leqq n-1 .
\end{aligned}
$$

Here we set

$$
\begin{aligned}
{ }_{0}^{l} \hat{B}_{i j}{ }^{k}= & -\frac{\lambda}{\sqrt{1+\lambda^{2}}}\left(2^{l} \xi_{i} \delta_{j k}+{ }^{l} \xi_{j} \delta_{i k}+{ }^{l} \xi_{k} \delta_{i j}\right) \\
& -\frac{\lambda}{\sqrt{1+\lambda^{2}}}\left(\sum_{p, q=1}^{n-1}{ }^{l} \xi_{p} \hat{A}_{i p}{ }^{q} \hat{A}_{j k}{ }^{q}\right) \quad \text { for } \quad 1 \leqq i, j, k, l \leqq n-1 .
\end{aligned}
$$


Then by (3.13) and (3.14) we have ${ }_{0}^{l} \hat{B}=\left({ }_{0}^{l} \hat{B}_{i j}{ }^{k}\right)_{1 \leqq i, j, k \leqq n-1} \in\left(\overline{\mathrm{g}}_{n-1}\right)_{\hat{A}}$ for $1 \leqq l \leqq n-1$ and ${ }^{l} \hat{B}_{i j}{ }^{k}-{ }_{0}^{l} \hat{B}_{i j}{ }^{k}={ }^{i} \hat{B}_{l j}{ }^{k}-{ }_{0}^{i} \hat{B}_{l j}{ }^{k}$ for $1 \leqq i, j, k, l \leqq n-1$. Hence if we set

$$
{ }^{l} \hat{C}_{i j}{ }^{k}={ }^{l} \hat{B}_{i j}{ }^{k}-{ }_{0}^{l} \hat{B}_{i j}{ }^{k} \quad \text { for } \quad 1 \leqq i, j, k, l \leqq n-1,
$$

then $\hat{C}=\left({ }^{l} \hat{C}_{i j}{ }^{k}\right)_{1 \leqq i, j, k, l \leqq n-1} \in\left(\overline{\mathfrak{g}}_{n-1}^{(1)}\right)_{\hat{A}}$. By these arguments we know that any $C \in\left(\overline{\mathfrak{g}}_{n}^{(1)}\right)_{\varphi(\lambda, \hat{A})}$ can be completely determined by ${ }^{n} \eta \in \boldsymbol{R}, \hat{C} \in\left(\overline{\mathfrak{g}}_{n-1}^{(1)}\right)_{\hat{A}}$ and ${ }^{n} \boldsymbol{\xi}=\left({ }^{n} \xi_{1}, \ldots,{ }^{n} \xi_{n-1}\right) \in \boldsymbol{R}^{n-1}$. Conversely it is clear that these variables are independent. Hence we have

$$
\operatorname{dim}\left(\overline{\mathfrak{g}}_{n}^{(1)}\right)_{\varphi(\lambda, \hat{A})}=1+\frac{1}{2} n(n-1)+n-1=\frac{1}{2} n(n+1) .
$$

This completes the proof the of the proposition.

Q.E.D.

\section{§4. The proof of Theorem 3.1}

As in the previous section, we shall fix an orthnormal basis $\left\{e_{a}\right\}_{0 \leqq a \leqq n}$ (resp. $\left\{v_{i}\right\}_{1 \leqq i \leqq n}$ ) of $\boldsymbol{T}$ (resp. $\left.\boldsymbol{N}\right)$. As usual every $\sigma \in \mathfrak{o}(\boldsymbol{T})$ (resp. $\rho \in \mathfrak{o}(\boldsymbol{N})$ ) can be represented by a skew symmetric matrix $\left(\sigma_{a}{ }^{b}\right)_{0 \leqq a, b \leqq n}\left(\operatorname{resp} .\left(\rho_{i}{ }^{j}\right)_{1 \leqq i, j \leqq n}\right)$ with respect to $\left\{e_{a}\right\}$ (resp. $\left\{v_{i}\right\}$ ).

Let $A \in \overline{\mathscr{G}}_{n}$. By $\alpha_{A}$, we mean the element of $\dot{\mathscr{G}}$ corresponding to $A$. Let us define a map $\psi: \mathfrak{o}(N) \times \overline{\mathscr{G}}_{n} \times R^{n} \rightarrow S^{2} T^{*} \otimes N$ by

$$
\psi(\rho, A, \tau)=\exp \rho \alpha_{A}{ }^{\exp \tau} \quad \text { for }(\rho, A, \tau) \in \mathfrak{o}(N) \times \overline{\mathscr{G}}_{n} \times \boldsymbol{R}^{n},
$$

where for each $\tau=\left(\tau_{1}, \cdots, \tau_{n}\right)$ we denote by $\tilde{\tau}$ the element of $\mathfrak{o}(T)$ such that

$$
\tilde{\tau}=\left(\begin{array}{cc}
0, \tau_{1}, \cdots, \tau_{n} \\
-\tau_{1} & \\
\vdots & 0 \\
-\tau_{n} &
\end{array}\right) .
$$

Note that since $\alpha_{A} \in \dot{\mathscr{G}}$, we have $\psi(\rho, A, \tau) \in \mathscr{G}$. Let us denote by $\psi_{*}=\psi_{*(0, A, 0)}$ the differential of $\psi$ at $(0, A, 0) \in \mathfrak{o}(N) \times \overline{\mathscr{G}}_{n} \times \boldsymbol{R}^{n}$, where 0 means the zero matrix of degree $n$ or the vector $(0, \cdots, 0) \in \boldsymbol{R}^{n}$. Naturally the tangent space to the variety $\mathfrak{o}(N) \times \overline{\mathscr{G}}_{n} \times \boldsymbol{R}^{n}$ at $(0, A, 0)$ may be identified with the direct sum $\mathfrak{o}(N) \oplus\left(\overline{\mathfrak{g}}_{n}\right)_{A} \oplus \boldsymbol{R}^{n}$. Hence $\psi_{*}$ induces a linear map of $\mathfrak{D}(N) \oplus\left(\overline{\mathrm{g}}_{n}\right)_{A} \oplus R^{n}$ into $S^{2} T^{*} \otimes N$. We also denote it by $\psi_{*}$.

Let $\rho=\left(\rho_{i}{ }^{j}\right)_{1 \leqq i, j \leqq n} \in \mathfrak{D}(N), B=\left(B_{i j}{ }^{k}\right)_{1 \leqq i, j, k \leqq n} \in\left(\overline{\mathrm{g}}_{n}\right)_{A}$, and $\tau=\left(\tau_{1}, \cdots, \tau_{n}\right) \in \boldsymbol{R}^{n}$. It is noted that the vectors $\psi_{*}(\rho), \psi_{*}(B)$ and $\psi_{*}(\tau)$ in $S^{2} T^{*} \otimes N$ are necessarily contained in $g_{\alpha_{A}}$. In terms of coefficients they are represented as follows:

$$
\begin{aligned}
& \psi_{*}(\rho)_{00}{ }^{k}=0, \quad \psi_{*}(\rho)_{0 i}{ }^{k}=\delta_{i k}, \quad \psi_{*}(\rho)_{i j}{ }^{k}=\sum_{l=1}^{n} \rho_{l}{ }^{k} A_{i j}{ }^{l} ; \\
&(* * *) \quad \psi_{*}(B)_{00}{ }^{k}=\psi_{*}(B)_{0 i}{ }^{k}=0, \quad \psi_{*}(B)_{i j}{ }^{k}=B_{i j}{ }^{k} ; \\
& \\
& \psi_{*}(\tau)_{00}{ }^{k}=-2 \tau_{k}, \quad \psi_{*}(\tau)_{0 i}{ }^{k}=-\sum_{l=1}^{n} \tau_{l} A_{l i}{ }^{k}, \quad \psi_{*}(\tau)_{i j}{ }^{k}=\tau_{i} \delta_{j k}+\tau_{j} \delta_{i k}
\end{aligned}
$$

for $1 \leqq i, j, k \leqq n$. 
Lemma 4.1. For each $\beta \in \mathfrak{g}_{a_{A}}$, there exists a unique $(\rho, B, \tau) \in \mathfrak{v}(N) \oplus\left(\overline{\mathfrak{g}}_{n}\right)_{A} \oplus \boldsymbol{R}^{n}$ such that

$$
\beta=\psi_{*}(\rho)+\psi_{*}(B)+\psi_{*}(\tau) .
$$

Proof. We first assume that $\psi_{*}(\rho)+\psi_{*}(B)+\psi_{*}(\tau)=0$ for some $(\rho, B, \tau) \in \mathfrak{o}(N)$ $\oplus\left(\bar{g}_{n}\right)_{A} \oplus \boldsymbol{R}^{n}$. Then by $(* * *)$, we immediately have $\rho=B=\tau=0$. This proves the uniqueness. We next show the decomposition. Let $\beta \in \mathfrak{g}_{\alpha_{A}}$ and let $\widetilde{B}=\left(\widetilde{B}_{a b}{ }^{k}\right)_{0 \leq a, b \leq n}$ $\in \boldsymbol{R}^{n(n+1)^{2}}$ be the coefficients of $\beta$. Take $\tau=\left(\tau_{1}, \cdots, \tau_{n}\right) \in \boldsymbol{R}^{n}$ so that $\tau_{k}=-\frac{1}{2} \widetilde{B}_{00}{ }^{k}$ for $1 \leqq k \leqq n$. We set $\bar{\beta}=\beta-\psi_{*}(\tau)$. Since $\bar{\beta} \in g_{\alpha_{A}}$, we have $\Omega_{* \alpha_{A}}(\bar{\beta})=0$, i.e.,

$$
\begin{gathered}
\left\langle\bar{\beta}(x, z), \alpha_{A}(y, w)\right\rangle+\left\langle\alpha_{A}(x, z), \bar{\beta}(y, w)\right\rangle-\left\langle\bar{\beta}(x, w), \alpha_{A}(y, z)\right\rangle \\
-\left\langle\alpha_{A}(x, w), \bar{\beta}(y, z)\right\rangle=0 \quad \text { for } \quad x, y, z, w \in T .
\end{gathered}
$$

Let $\left(\bar{B}_{a b}{ }^{k}\right)_{\substack{0 \leqq a, b \leqq n \\ 1 \leqq k \leqq n}}$ be the coefficients of $\bar{\beta}$. By the choice of $\tau$, we have $\bar{B}_{00}{ }^{k}=0$ for $1 \leqq i \leqq n$. Thus if we put $x=e_{0}, y=e_{i}, z=e_{0}, w=e_{j}(1 \leqq i, j \leqq n)$ into (4.1), then we obtain $\bar{B}_{0 i}{ }^{j}+\bar{B}_{0 j}{ }^{i}=0$. Let us set $\rho=\left(\bar{B}_{0 i}{ }^{j}\right)_{1 \leqq i, j \leqq n}$ and set $\stackrel{\beta}{\beta}=\bar{\beta}-\psi_{*}(\rho)=\beta-\left(\psi_{*}(\tau)\right.$ $\left.+\psi_{*}(\rho)\right)$. Since $\rho \in \mathfrak{D}(N)$, we have $\dot{\beta} \in \mathrm{g}_{\alpha_{A}}$. Hence

$$
\begin{gathered}
\left\langle\dot{\beta}(x, z), \alpha_{A}(y, w)\right\rangle+\left\langle\alpha_{A}(x, z), \dot{\beta}(y, w)\right\rangle-\left\langle\dot{\beta}(x, w), \alpha_{A}(y, z)\right\rangle \\
-\left\langle\alpha_{A}(x, w), \dot{\beta}(y, z)=0 \quad \text { for } \quad x, y, z, w \in T .\right.
\end{gathered}
$$

Let $\stackrel{\circ}{B}=\left(\check{B}_{a, b}{ }^{k}\right)_{\substack{0 \leqq a, b \leqq n \\ 1 \leqq k \leqq n}}$ be the coefficients of $\dot{\beta}$. Then we have $\stackrel{B}{B}_{00}{ }^{k}=\stackrel{B}{B}_{0 i}{ }^{k}=0$ for $1 \leqq i$, $k \leqq n$. By this relation, the equation (4.2) may be reduced to the system of equations:

$$
\begin{aligned}
& \dot{B}_{i j}{ }^{k}=\dot{B}_{i k}{ }^{j} ; \quad \sum_{p=1}^{n}\left(\dot{B}_{i k}{ }^{p} A_{j l}{ }^{p}+A_{i k}{ }^{p} \dot{B}_{j l}{ }^{p}-\dot{B}_{i l}{ }^{p} A_{j k}{ }^{p}-A_{i}^{l p} \dot{B}_{j k}{ }^{p}\right)=0 \\
& \text { for } 1 \leqq i, j, k, l \leqq n \text {. }
\end{aligned}
$$

Thus if we set $B=\left(\dot{B}_{i j}{ }^{k}\right)_{1 \leqq i, j, k \leqq n}$, we have $B \in\left(\bar{g}_{n}\right)_{A}$ and $\dot{\beta}^{\prime}=\psi_{*}(B)$. Hence we have $\beta=\psi_{*}(\rho)+\psi_{*}(B)+\psi_{*}(\tau)$.

Q.E.D.

These being prepared, we start the proof of Theorem 3.1. Let $U$ be the open set in $\boldsymbol{R}^{n}$ stated in Proposition 3.2. Let $A_{0} \in \overline{\mathscr{G}}_{n} \cap U$. By Lemma 4.1. we know that the differential $\psi_{*}$ of $\psi_{\text {at }}\left(0, A_{0}, 0\right)$ is injective. Taking a sufficiently small open neighborhood $\widetilde{O}$ of $\left(0, A_{0}, 0\right)$ in $\mathrm{D}(N) \times\left(\bar{G}_{n} \cap U\right) \times \boldsymbol{R}^{n}$, we may assume that the restriction of the map $\psi$ to $\widetilde{O}$ is an imbedding. Therefore the image $\psi(\widetilde{O})$ of $\widetilde{O}$ forms an $n(n-1)$-dimensional submanifold of $S^{2} T^{*} \otimes N$. Note that $\overline{\mathscr{G}}_{n} \cap U$ is a $\frac{1}{2} n(n-1)$ dimensional submanifold of $R^{n^{3}}$. Moreover we know $\mathrm{g}_{\alpha_{A_{0}}}=T_{\alpha_{A_{0}}} \psi(\tilde{O})$. Hence by Lemma 3.4, there exists an open neighborhood $O$ of $\alpha_{A_{0}}$ in $S^{2} T^{*} \otimes N$ such that $\mathscr{G} \cap O$ $=\psi(\widetilde{O}) \cap O$. This shows (1) of the theorem.

Next we show (2) of the theorem. Let $\alpha \in \mathscr{G} \cap O$. Since $\alpha \in \psi(\widetilde{O})$, we may write $\alpha=\psi(\rho, A, \tau)$ by using suitable $(\rho, A, \tau) \in \mathfrak{o}(N) \times\left(\mathscr{G}_{n} \cap U\right) \times \boldsymbol{R}^{n}$. Hence in order to show (2), it suffices to deal with the case where $\alpha=\psi(0, A, 0)=\alpha_{A}$. Since $A \in \overline{\mathscr{G}}_{n} \cap U$ there exists a vector $x=\left(x_{1}, \cdots, x_{n}\right) \in \boldsymbol{R}^{n}$ satisfying (2) of Proposition 3.2. Here we may assume that $\left|x_{i}\right| \ll 1$ for $1 \leqq i \leqq n$. We set $e=e_{0}+\sum_{i=1}^{n} x_{i} e_{i}$. Then 
we can see that the vector $e$ satisfies the condition in (2). In fact, let us suppose $e\rfloor \beta=0$ for some $\beta \in g_{\alpha_{A^{*}}}$. Then by using the decomposition $\beta=\psi_{*}(\rho)+\psi_{*}(B)+$ $\psi_{*}(\tau)$ in Lemma 4.1. and the formulas $(* * *)$, we have

$$
\begin{gathered}
-2 \tau_{k}+\sum_{i=1}^{n} x_{i}\left(\rho_{i}{ }^{k}-\sum_{j=1}^{n} \tau_{j} A_{j i}{ }^{k}\right)=0 \quad \text { for } \quad 1 \leqq k \leqq n ; \\
\rho_{i}{ }^{k}-\sum_{j=1}^{n} \tau_{j} A_{j i}{ }^{k}+\sum_{j=1}^{n} x_{j}\left(\tau_{j} \delta_{i k}+\tau_{i} \delta_{j k}+\sum_{l=1}^{n} \rho_{l}{ }^{k} A_{j i}{ }^{l}\right) \\
+\sum_{j=1}^{n} x_{j} B_{j i}{ }^{k}=0 \quad \text { for } 1 \leqq i, k \leqq n .
\end{gathered}
$$

The equation (4.3) and the skew symmetric part of (4.4) with respect to the pair $(i, k)$ form a system of homogeneous linear equations with variables $\tau_{i}, \rho_{j}{ }^{k}(1 \leqq i, j, k \leqq n)$. Since $\left|x_{i}\right| \ll 1$ it follows that $\tau_{i}=\rho_{j}{ }^{k}=0$ for $1 \leqq i, j, k \leqq n$. Hence we have $\sum_{j=1}^{n} x_{j} B_{j i}{ }^{k}$ $=0$ for any $1 \leqq i, k \leqq n$. Therefore we obtain $B_{j i}{ }^{k}=0$ for any $1 \leqq i, j, k \leqq n$. This shows $\beta=0$.

Finally we show (3) of the theorem. As in the proof of (2), we may assume that $\alpha=\alpha_{A}$. Let $\gamma \in \mathfrak{g}_{\alpha_{A}}^{(1)}$. Since $\left.e_{s}\right\lrcorner \gamma \in \mathfrak{g}_{\alpha_{A}}$ for $0 \leqq s \leqq n$, there are ${ }^{s} \rho=\left({ }^{s} \rho_{i}{ }^{j}\right)_{1 \leqq i, j \leqq n} \in \mathfrak{o}(N)$, ${ }^{s} B=\left({ }^{s} B_{i j}{ }^{k}\right)_{1 \leqq i, j, k \leqq n} \in\left(\bar{g}_{n}\right)_{A}$ and ${ }^{s} \tau=\left({ }^{s} \tau_{1}, \cdots,{ }^{s} \tau_{n}\right) \in R^{n}$ such that $\left.e_{s}\right\rfloor \gamma=\Psi_{*}\left({ }^{s} \rho\right)+$ $\Psi_{*}\left({ }^{s} B\right)+\Psi_{*}\left({ }^{s} \tau\right)$. Then, by the relation $\left.\left.\left.\left.e_{t}\right\rfloor e_{s}\right\rfloor \gamma=e_{s}\right\rfloor e_{t}\right\rfloor \gamma$ for $1 \leqq s, t \leqq n$, we have

$$
\begin{aligned}
& { }^{i} \tau_{k}=\frac{1}{2}{ }^{0} \rho_{k}{ }^{i}+\frac{1}{2} \sum_{j=1}^{n}{ }^{0} \tau_{j} A_{j k}{ }^{i} \quad \text { for } \quad 1 \leqq i, k \leqq n ; \\
& { }^{i} \rho_{j}{ }^{k}-\sum_{l=1}^{n}{ }^{i} \tau_{l} A_{l j}{ }^{k} \\
& ={ }^{0} \tau_{i} \delta_{j k}+{ }^{0} \tau_{j} \delta_{i k}+\sum_{l=1}^{n}{ }^{0} \rho_{l}{ }^{k} A_{i j}{ }^{l}+{ }^{0} B_{i j}{ }^{k} \quad \text { for } \quad 1 \leqq i, j, k \leqq n ; \\
& { }^{l} B_{i j}{ }^{k}-{ }^{i} B_{l j}{ }^{k}=\frac{1}{2}\left({ }^{0} \rho_{l}{ }^{i} \delta_{j k}+{ }^{0} \rho_{l}{ }^{j} \delta_{i k}+{ }^{0} \rho_{l}{ }^{k} \delta_{j i}\right)-\frac{1}{2}\left({ }^{0} \rho_{i}{ }^{l} \delta_{j k}+{ }^{0} \rho_{i}{ }^{j} \delta_{l k}+{ }^{0} \rho_{i}{ }^{k} \delta_{j l}\right) \\
& +\frac{1}{2} \sum_{p, q=1}^{n}{ }^{0} \rho_{p}^{q}\left(A_{l k}{ }^{p} A_{i j}{ }^{q}-A_{i k}{ }^{p} A_{l j}{ }^{q}\right) \quad \text { for } \quad 1 \leqq i, j, k, l \leqq n \text {. }
\end{aligned}
$$

From (4.6) we obtain

$$
\begin{aligned}
& { }^{i} \rho_{j}{ }^{k}=\frac{1}{2}\left({ }^{0} \tau_{j} \delta_{i k}-{ }^{0} \tau_{k} \delta_{i j}\right)+\frac{1}{2} \sum_{l=1}^{n}\left({ }^{0} \rho_{l}{ }^{k} A_{i j}{ }^{l}-{ }^{0} \rho_{l}{ }^{j} A_{i k}{ }^{l}\right) ; \\
& { }^{0} B_{i j}{ }^{k}=-\frac{1}{2}\left({ }^{0} \tau_{j} \delta_{i k}+{ }^{0} \tau_{k} \delta_{i j}+2{ }^{0} \tau_{i} \delta_{j k}\right)-\frac{1}{2} \sum_{p, q=1}^{n}{ }^{0} \tau_{q} A_{q i}{ }^{p} A_{j k}{ }^{p} \\
& -\frac{1}{2} \sum_{p=1}^{n}\left({ }^{0} \rho_{p}{ }^{k} A_{i j}{ }^{p}+{ }^{0} \rho_{p}{ }^{j} A_{i k}{ }^{p}+{ }^{0} \rho_{p}{ }^{i} A_{p j}{ }^{k}\right) \quad \text { for } \quad 1 \leqq i, j, k \leqq n .
\end{aligned}
$$

We set

$$
\begin{aligned}
{ }_{0}^{l} B_{i j}{ }^{k} & =-\frac{1}{2}\left({ }^{l} \tau_{j} \delta_{i k}+{ }^{l} \tau_{k} \delta_{i j}+2{ }^{l} \tau_{i} \delta_{j k}\right)-\frac{1}{2} \sum_{p, q=1}^{n}{ }^{l} \tau_{q} A_{q i}{ }^{p} A_{j k}{ }^{p} \\
& -\frac{1}{2} \sum_{p=1}^{n}\left({ }^{l} \rho_{p}{ }^{k} A_{i j}{ }^{p}+{ }^{l} \rho_{q}{ }^{j} A_{i k}{ }^{p}+{ }^{l} \rho_{p}{ }^{i} A_{p j}{ }^{k}\right) \quad \text { for } \quad 1 \leqq i, j, k, l \leqq n .
\end{aligned}
$$


Then we have ${ }_{0}^{l} B=\left({ }_{0}^{l} B_{i j}{ }^{k}\right)_{1 \leqq i, j, k \leqq n} \in\left(\bar{g}_{n}\right)_{A}$ for $1 \leqq l \leqq n$. Moreover by (4.5), (4.7) and (4.8) we obtain ${ }^{l} B_{i j}{ }^{k}-{ }_{0}^{l} B_{i j}{ }^{k}={ }^{i} B_{l j}{ }^{k}-{ }_{0}^{i} B_{l j}{ }^{k}$ for $1 \leqq i, j, k, l \leqq n$. Let us set

$$
{ }^{l} C_{i j}{ }^{k}={ }^{l} B_{i j}{ }^{k}-{ }_{0}^{l} B_{i j}{ }^{k} \quad \text { for } 1 \leqq i, j, k, l \leqq n .
$$

Then we have $C=\left({ }^{l} C_{i j}{ }^{k}\right)_{1 \leqq i, j, n, l \leqq n} \in\left(\overline{\mathfrak{g}}_{n}^{(1)}\right)_{A}$. Therefore any $\gamma \in \mathfrak{g}_{\alpha_{A}}^{(1)}$ can be completely determined by ${ }^{0} \rho==\left({ }^{0} \rho_{i}{ }^{j}\right)_{1 \leqq i, j \leqq n} \in \mathrm{D}(N), C=\left({ }^{l} C_{i j}{ }^{k}\right)_{1 \leqq i, j, k, l \leqq n} \in\left(\overline{\mathfrak{g}}_{n}^{(1)}\right)_{A}$ and ${ }^{0} \tau=\left({ }^{0} \tau_{1}, \cdots\right.$, $\left.{ }^{0} \tau_{n}\right) \in \boldsymbol{R}^{n}$. Conversely it is clear that these variables are independent. Hence we have $\operatorname{dim} g_{\alpha_{A}}^{(1)}=\frac{1}{2} n(n-1)+\frac{1}{2} n(n+1)+n=n(n+1)$. Thus we have completed the proof of Theorem 3.1 .

\section{§5. Isometric immersions of the spaces of negative constant curvature}

Let $(M, g)$ be the space of constant curvature of dimension $n$ with sectional curvature $k<0$. Then at each $p \in M$, we have

$$
\begin{array}{r}
-g(R(x, y) z, w)=k\{g(x, z) g(y, w)-g(x, w) g(y, z)\} \\
\text { for } x, y, z, w \in T_{p} .
\end{array}
$$

Moreover we have $\nabla R \equiv 0$ on $M$.

We now show the following

Theorem 5.1. If $m=2 n-1$, then there exists an open fibered submanifold $\pi_{1}^{2}: P_{\#}^{(1)}$ $\rightarrow P$ of the vector bundle $\pi_{1}^{2}: P^{(1)} \rightarrow P$ such that the intersection $Q_{\sharp}=Q \cap P_{\#}^{(1)}$ forms an involutive differential equation. ${ }^{(* *)}$

Proof. Let $\alpha \in P$ and let $V$ be a sufficiently small neighborhood of $\alpha$ in $P$. From the local triviality of the vector bundle $\pi: N \rightarrow P$, we may assume that $\pi^{-1}(V)$ is isomorphic to the vector bundle $V \times N$, where $N=N_{\alpha}$. Furthermore we may assume that the isomorphism gives an isometric isomorphism between each fiber of $\pi^{-1}(V)$ and $N$. (Note that since each fiber of the vector bundle $\pi: N \rightarrow P$ is a subspace of $R^{2 n-1}$, it is endowed with an inner product.) Similarly the restriction $T_{\mid \pi_{-1}^{1}(v)}$ of the tangent bundle $T=T(M)$ to $\pi_{-1}^{1}(V)$ may be assumed to be isomorphic to the bundle $\pi_{-1}^{1}(V) \times T$, where we set $T=T_{p}$. We may assume that the isomorphism gives an isometric isomorphism between each fiber of $T_{\mid \pi_{-1}^{1}(v)}$ and $T$ with respect to the given Riemannian metric $g$. Under these observations the restriction $P_{\mid v}^{(1)}$ of the vector bundle $\pi_{1}^{2}: P^{(1)} \rightarrow P$ to $V$ may be considered to be isomorphic to the bundle $V \times S^{2} T^{*} \otimes N$. By this isomorphism the set $Q \cap\left(\pi_{1}^{2}\right)^{-1}(V)$ is mapped onto $V \times \mathscr{G}\left(G_{k}\right)$ (see the equation (1.8)). Hence there exists an open set $O$ in $S^{2} T^{*} \otimes N$ having the property stated in Theorem 3.1. By $O_{\alpha}$ we denote the open set in $P^{(1)}$ that corresponds to the open set $V \times O$ in $V \times S^{2} T^{*} \otimes N$. For any $\alpha \in P$, we take such an open set $O_{\alpha}$ in $P^{(1)}$ and set $P_{\sharp}^{(1)}=\bigcup_{\alpha \in P} O_{\alpha}$. Then it is clear that $\pi_{1}^{2}: P_{\sharp}^{(1)} \rightarrow P$ forms an open fibered submanifold of the vector bundle $\pi_{1}^{2}: P^{(1)} \rightarrow P$. We set $Q_{\sharp}=Q \cap P_{\sharp}^{(1)}$ and

(**) For the definition of involutive differential equations, see [8] or [4]. 
$Q_{\#}^{(1)}=Q^{(1)} \cap\left(\pi_{2}^{3}\right)^{-1}\left(P_{\#}^{(1)}\right)$. Then we can easily verify that $Q_{\#}$ forms a fibered submanifold of the vector bundle $\pi_{1}^{2}: P^{(1)} \rightarrow P$. Hence $Q_{\#}$ is a differential equation. In order to show the involutiveness of $Q_{\#}$, we must show the following:

(a) The map $\pi_{2}^{3}: Q_{\sharp}^{(1)} \rightarrow Q_{\sharp}$ is surjective.

(b) The union $\mathfrak{q}^{(1)}=\bigcup_{\beta \in Q_{\sharp}} \mathfrak{q}_{\beta}^{(1)}$ is a vector bundle over $Q_{\#}$.

(c) For each $\beta \in Q_{\sharp}$, the symbol $\mathrm{q}_{\beta}$ of $Q_{\sharp}$ at $\beta$ is involutive.

Proof of $(a)$. Let $\beta=\left(p ; \omega_{0}, \omega_{1}, \omega_{2}\right) \in Q_{\#} . \quad$ Let us define $\bar{\omega}_{3} \in \otimes^{3} T_{p}^{*} \otimes R^{2 n-1}$ by setting

$$
\begin{array}{ll}
\left\langle\bar{\omega}_{3}(w, z, x), \omega_{1}(y)\right\rangle=-\left\langle\omega_{2}(z, x), \omega_{2}(w, y)\right\rangle, \\
\left\langle\bar{\omega}_{3}(w, z, x), n\right\rangle=0 \quad \text { for } x, y, z, w \in T_{p}, n \in N_{\alpha}\left(\alpha=\pi_{1}^{2}(\beta)\right) .
\end{array}
$$

Then we have $\gamma=\left(p ; \omega_{0}, \omega_{1}, \omega_{2}, \bar{\omega}_{3}\right) \in J^{3}(M, m)$ and $\pi_{2}^{3}(\gamma)=\beta$. Moreover since $\nabla R \equiv 0$ we have $\gamma \in Q_{\sharp}^{(1)}$ (see the equations (1.9) and (1.10)). Hence the map $\pi_{2}^{3}: Q_{\#}^{(1)} \rightarrow Q_{\#}$ is surjective.

Proof of $(b)$ and $(c)$. Let $\beta=\left(p ; \omega_{0}, \omega_{1}, \omega_{2}\right) \in Q_{\#}$. We set $\alpha=\pi_{1}^{2}(\beta)$. We may assume $\beta \in O_{\alpha}$. Then by the definitions of the vector spaces $\mathfrak{q}_{\beta}$ and $\mathfrak{q}_{\beta}^{(1)}$, we have $\mathfrak{q}_{\beta}$ $=\mathfrak{g}_{\omega_{2}}$ and $\mathfrak{q}_{\beta}^{(1)}=\mathfrak{g}_{\omega_{2}}^{(1)}$ (Note that we are assuming $T=T_{p}$ and $N=N_{\alpha}$.) Since $\operatorname{dim} N=$ $\operatorname{dim} T-1=n-1$, we have $\operatorname{dim} \mathfrak{q}_{\omega_{2}}^{(1)}=\operatorname{dim} \mathfrak{g}_{\omega_{2}}^{(1)}=n(n-1)$. This indicates that the union $\mathfrak{q}^{(1)}=\bigcup_{\beta \in Q_{*}} \mathfrak{q}_{\beta}^{(1)}$ is a vector bundle over $Q_{*}$. We next show (c). Since $\beta \in O_{\alpha}$, there exists a vector $e \in T=T_{p}$ such that $\left.e\right\rfloor \xi \neq 0$ for any $\xi \in \mathfrak{q}_{\beta} ; \xi \neq 0$. Then we can easily see that any basis $\left\{e_{1}, \cdots, e_{n}\right\}$ of $\boldsymbol{T}=T_{p}$ such that $e_{1}=e$ is regular for the symbol $q_{\beta}$. Hence $\mathfrak{q}_{\beta}$ is involutive. Thus we have completed the proof of Theorem 5.1. Q.E.D.

Note that any space of constant curvature is a real analytic Riemanian manifold. Then the varieties $P, P^{(1)}, Q^{(1)}$ and $Q$ are also considered to be real analytic. We can easily see that Theorem 5.1 still holds if we consider everything in the real analytic category. Then $Q_{\#}$ forms a real analytic differential equation. From the existence theroem of local solutions of real analytic involutive differential equations (cf. [8], [4]) follows

Theorem 5.2. Any space of negative constant curvature of dimension $n$ can be locally isometrically immersed into the euclidean space $\boldsymbol{R}^{2 n-1}$.

\section{Department of Mathematics, KYOTO UNIVERSITY}

\section{Bibliography}

[ 1 ] D. Blanuša, Úber die Einbettung hyperbolischer Räume in euklidische Räume, Monatsch. Math. 59 (1955), 217-229.

[2] E. Cartan, Sur la possibilité de plonger un espace riemannien donné dans un espace euclidien, Ann. Soc. Pol. Math. 6 (1927), 1-7.

[ 3 ] J. Gasqui, Sur l'existence d'immersions isometriques locales pour les variétés riemanniens, J. Diff. Geom. 10 (1975), 61-84. 
[ 4 ] H. Goldschmidt, Integrability criteria for systems of non linear partial differential equations, J. Diff. Geom. 6 (1972), 357-373.

[ 5 ] M. Janet, Sur la possibilité de plonger un espace riemannien donné dans un espace euclidien, Ann. Soc. Pol. Math. 5 (1926), 38-43.

[6] E. Kaneda and N. Tanaka, Rigidity for isometric imbeddings, to appear in J. Math. Kyoto Univ.

[ 7 ] S. Kobayashi and K. Nomizu, Foundations of differential geometry II, Wiley-Interscience, New York (1969).

[ 8 ] M. Kuranishi, Lectures on involutive systems of partial differential equations, Publ. Soc. Mat. São Paulo (1967).

[9] T. Otsuki, Isometric imbedding of Riemannian manifolds in a Riemannian manifold, J. Math. Soc. Japan 6 (1954), 221-234.

[10] N. Tanaka, Rigidity for elliptic isometric imbeddings, Nagoya Math. J. 51 (1973), $137-160$.

Added in proof: After submitting this paper, the author knew the following classical work:

E. Cartan, Sur les variétés de courbure constante d'un espace euclidien ou noneuclidean, Oeuvres complètes, Partie III, vol. 1, Gauthier-Villars, Paris, 1955. 